\title{
A NOTE ON QUASI-METRIC SPACES ${ }^{1}$
}

\author{
GEORGE E. ALBERT
}

1. Introduction. In a recent abstract ${ }^{2}$ the author introduced an important application of a class of neighborhood spaces in which pairs of points are required to satisfy only a very weak separation axiom (due to Kolmogoroff):

(K) If $x$ and $y$ are distinct points, then at least one of these points has a neighborhood which does not contain the other.

In the present note it is shown that a very useful generalized distance function may be defined in certain of these spaces.

Clearly, any such distance function must be an asymmetric one. W. A. Wilson ${ }^{3}$ considered the definition of asymmetric distances in certain spaces which satisfy stronger separation axioms than $\mathrm{K}$. It is shown here that a slight modification of one of the axioms in [W] allows the extension of a large part of the theory developed there to spaces subject to $\mathrm{K}$.

Since many of the theorems and proofs in [W] remain valid here with only very obvious changes, this note will be limited to a mere sketch concerning new properties which arise under the weaker axioms used. The reader should have no difficulty in adapting the more complete discussion given in $[\mathrm{W}]$ to the case studied here.

2. The distance function. The symbol 1 will denote a space of points; points and point sets in 1 will be denoted by small and capital letters respectively.

The space 1 will be said to be quasi-metric if for every pair of points $x, y$ in 1 there are defined two non-negative numbers $x y$ and $y x$, not necessarily equal, which satisfy the following postulates:

I. $x y=y x=0$ if and only if $x=y ; 4$

II. for any three points, $x y \leqq x z+z y$.

If for some pair of points $x y=0 \neq y x$, then the point $x$ will be said to be adjacent to the point $y$. It is to be emphasized that, regardless

\footnotetext{
1 Presented to the Society, April 13, 1940.

2 Abstract 46-3-138, this Bulletin.

${ }^{3}$ W. A. Wilson, On quasi-metric spaces, American Journal of Mathematics, vol. 53 (1931), p. 675. Hereafter this paper will be referred to as [W].

${ }^{4}$ In [W] the stronger axiom: $x y=0$ if and only if $x=y$ was used. This excludes the case $x y=0 \neq y x$ allowed here.
} 
of the connotation of symmetry familiar with the word adjacent, the relationship to which it refers here is an asymmetric one.

3. Limits, closed and open sets. The nature of the function $x y$ leads to the definition of three separate types of limit points and the corresponding classes of closed and open sets. A point $p$ will be called a $u$-limit, $l$-limit, or a $c$-limit of a point set $A$ (of a sequence $\left\{x_{n}\right\}$ ) if for every positive number $\epsilon$ there exists a point $x$ in $A-p$ such that $p x<\epsilon, x p<\epsilon$, or both simultaneously, respectively (if there is a number $N$ such that $p x_{n}<\epsilon$, and so on, for $\left.n>N\right)$.

The point set $S_{u}(a, r)$ consisting of all points $x$ such that $a x<r$ will be called a $u$-sphere of center $a$ and radius $r$. Similarly one defines the spheres $S_{l}(a, r)$ and $S_{c}(a, r)$ by the relations $x a<r$ and both $a x<r$ and $x a<r$.

A point set will be termed $u$-open, l-open, or c-open if for every point $p$ in the set some sphere $S_{u}, S_{l}$, or $S_{c}$, respectively, about $p$ is contained entirely within the set. Such sets will be denoted by $G_{u}, G_{l}$, and $G_{c}$ respectively. A point set will be termed $u$-closed, $l$-closed, or c-closed if it contains all of its limit points of the respective class. These sets will be denoted by $F_{u}, F_{l}$, and $F_{c}$ respectively.

The space 1 will be called separable if there exists a denumerable sequence of points which is everywhere dense in 1 in the sense of $c$-limits.

Just as in the theory of ordinary topological spaces, one proves that the complement of a set $G_{u}\left(G_{l}\right.$ or $\left.G_{c}\right)$ is a set $F_{u}\left(F_{l}\right.$ or $\left.F_{c}\right)$. It is evident that every set $F_{u}$ or $F_{l}$ is also a set $F_{c}$ and that every set $G_{u}$ or $G_{l}$ is also a set $G_{c}$; however the converses are not true.

It is to be noted that a point $p$ may be a $u$-limit and at the same time an $l$-limit of a point set $A$ without being a $c$-limit of that set. On the other hand, for sequences one has the following theorem:

Theorem 1. If $x=u-\lim x_{n}$ and $x=l-\lim x_{n}$ then $x=c-\lim x_{n}$. Moreover, there exists no other c-limit of the sequence $\left\{x_{n}\right\}$ and the Cauchy criterion of convergence is satisfied by the sequence.

Proof. The first clause is immediate from the definitions.

Suppose that $x$ and $y$ are both $c$-limits of the sequence $\left\{x_{n}\right\}$. It follows that for an arbitrary positive number $\epsilon$ there exist numbers $n^{\prime}$ and $n^{\prime \prime}$ such that $x y \leqq x x_{n}+x_{n} y<\epsilon$ for $n>n^{\prime}$ and $y x \leqq y x_{n}+x_{n} x<\epsilon$ for $n>n^{\prime \prime}$. Thus $x y=0=y x$ and by I, $x=y$.

Again, $x=c$ - $\lim x_{n}$ implies that for arbitrary $\epsilon>0$ there is an $n^{\prime}$ such that $x_{n} x_{m} \leqq x_{n} x+x x_{m}<\epsilon$ for $n>n^{\prime}$ and the Cauchy criterion is satisfied. 
The following theorems indicate the relations between the several limits of a sequence and the property of adjacency.

Theorem 2. If $x=l-\lim x_{n}$ and $y=u$ - $\lim x_{n}$ for the same sequence $\left\{x_{n}\right\}$, then either $x=y$ or else $y$ is adjacent to $x$.

The proof is immediate from $y x \leqq y x_{n}+x_{n} x$ and the fact that $y x_{n} \rightarrow 0$ and $x_{n} x \rightarrow 0$.

COROLlaRy. If $x=c-\lim x_{n}$ and $y=u-\lim x_{n}$ then either $y=x$ or $y$ is adjacent to $x$; if $y=l-\lim x_{n}$, then either $y=x$ or $x$ is adjacent to $y$.

It is worthy of note that if a point $x$ is adjacent to a point $y$ then $x$ is a $u$-limit of the point set consisting of the single point $y$. Thus, if the usual definition of connected set be adopted with limits taken in the $u$-sense, the point set $(x+y)$ forms a connected set.

4. Topological versus quasi-metric spaces. Hausdorff $f^{5}$ stated the following axioms for topological spaces:

(A) Every point $p$ has a neighborhood $U_{p}$. For every $U_{p}, p \in U_{p}$.

(B) For every two neighborhoods $U_{p}$ and $V_{p}$ of the point $p$, there is a third $W_{p} \subset U_{p} V_{p}$.

(C) For every point $q$ in $U_{p}$, there is a neighborhood $U_{q} \subset U_{p}$.

(D) To each point $p$ there corresponds a denumerable set of neighborhoods such that every neighborhood $U_{p}$ contains a neighborhood of that set.

(E) There exists a denumerable set of neighborhoods $\left\{U_{n}\right\}$ such that if $U_{p}$ is any neighborhood of the point $p$, then there is an $n$ such that $p \in U_{n} \subset U_{p}$.

Theorem 3. Let 1 denote a quasi-metric space and, for each point $x$ in 1 , let the class of spheres $S_{u}(x, r)$ of rational radii $r$ be termed neighborhoods of $x$. Then 1 is a topological space satisfying the axioms A, B, C, $\mathrm{D}$, and $\mathrm{K}$. The u-limits of sequences of points in 1 are the topological limits of the sequences and conversely. If the space is separable, Axiom D may be replaced by the stronger Axiom $\mathrm{E}$. The theorem remains valid if the spheres $S_{u}$ are replaced by the spheres $S_{l}$ and u-limits by l-limits.

The proof is evident.

THEOREM 4. Let 1 be a neighborhood space satisfying the axioms $\mathrm{A}, \mathrm{B}, \mathrm{C}, \mathrm{E}$, and $\mathrm{K}$. Then distances can be defined in 1 so that the space is a separable, quasi-metric space. A topological limit of a sequence of

' Hausdorff, Mengenlehre, Berlin, 1935, pp. 228, 229. 
points in 1 is a u-limit of the sequence and conversely. The theorem remains valid if u-limit is replaced by l-limit provided that a corresponding change be made in the distance function.

Since the proof of this theorem is long but follows that of Theorem IV, $\S 7$, in [W] with only a few minor changes, it seems sufficient to state the definition of the distance function for the case of $u$-limits.

Let the denumerable system of neighborhoods postulated by Axiom $\mathrm{E}$ be denoted by $\left\{U_{n}\right\}$. For any pair of points $x$ and $y$ in 1 and for each $n=1,2,3, \cdots$, define the function $f_{n}(x, y)$ to be equal 1 if $x$ is in $U_{n}$ and $y$ is in $1-U_{n}$, or equal to 0 in case $x$ is in $1-U_{n}$ or in case $x+y$ is in $U_{n}$. Then define the function

$$
x y=\sum_{n=1}^{\infty} \frac{1}{2^{n}} f_{n}(x, y) \text {. }
$$

It should be remarked that a quasi-metric is not necessarily a continuous function of either variable.

5. Applications. The distance function described above furnishes an exceedingly simple topologization for a very general type of collection of closed point sets. Let $S$ denote a compact metric space and let $S=\sum X$ be any decomposition of $S$ into closed point sets. It is not assumed that the sets of the decomposition are disjoint. However it is assumed that if $X_{1}$ and $X_{2}$ are distinct elements of the decomposition which overlap, then $X_{1} X_{2}=X$ is again an element of the decomposition. Moreover, if $X_{3}$ is any element which intersects $X$, then $X_{1} X_{3}=X=X_{2} X_{3}$. Such a collection is afforded by the upper semicontinuous collection of the second type in troduced by R. L. Moore ${ }^{6}$ and, in particular, by the so-called hyperspace of cyclic elements of a continuous curve. If, now, the elements of the collection are thought of as points of a new space 1, the topologization is introduced as follows: if $X$ and $Y$ are two elements of 1 , the distance function $X Y=$ the greatest lower bound of the numbers $r$ such that, as seen in the space $S$, every point $x$ of the set $X$ has a distance from the set $Y$ which is less than $r$. The space is easily shown to be quasi-metric in the sense described in the preceding paragraphs.

Ohio State University

${ }^{6}$ The Rice Institute Pamphlet, vol. 23, no. 1, 1936, p. 42. 